\title{
THE COSMO-SKYMED SECOND GENERATION SAR ANTENNA ELECTRICAL POWER CHAIN AND PLATFORM POWER DISTRIBUTION
}

\author{
E. Scorzafava ${ }^{(1)}$ \\ G. Gianninoto ${ }^{(2)}$, M. Cantamessa ${ }^{(2)}$, P. Zanella ${ }^{(2)}$ \\ D. Carlani $^{(3)}$, E. Scione ${ }^{(3)}$ \\ (1) Italian Space Agency (ASI) - viale del Politecnico snc Roma (Italy),Email: edmondo.scorzafava@asi.it \\ (2)Leonardo S.p.A. - viale Europa Nerviano (Italy),Email:guido.gianninoto@leonardocompany.com, \\ marco.cantamessa@leonardocompany.com,pietro.zanella@leonardocompany.com, \\ (3) Thales Alenia Space Italia - via Saccomuro 24 - Roma (Italy), Email: daniele.carlani@thalesaleniaspace.com, \\ emiliano.scione@thalesaleniaspace.com
}

\begin{abstract}
This paper presents the Electrical power Subsystem (EPS) of the COSMO-SKYMED SECOND GENERATION (CSG) spacecraft, regarding the design and development of the electronics for power generation, conditioning and distribution. It provides a detailed description of the power equipment's and of the Photovoltaic Assembly (PVA).

The design characteristics of the EPS elements are described. A summary of the main performances is given, including a glance to the EMC aspects, which are very important in the definition and characterization of the design of a high power SAR system.

It will be also shown how the design of the power conditioning and distribution electronics has taken advantage of the Leonardo (former Finmeccanica) heritage on Power Management and Distribution (PMD) for scientific platforms [1], [3].
\end{abstract}

\section{COSMO SECOND GENERATION MISSION}

COSMO-SKYMED SECOND GENERATION (CSG) is a program founded by the Italian Space Agency (ASI) and Minister of Defense (MoD) aimed to earth observation by means of a Synthetic Aperture Radar (SAR) Antenna. Its mission has been defined to continue the operation of the four spacecrafts fleet operated in the frame of the COSMO SkyMed program first generation.

The present program consists of two satellites. The first satellite launch is foreseen within 2018 , the second one within 2019.

Each satellite will carry a Synthetic Aperture Radar Antenna (SAR) of new generation, to perform the observation of the earth. Its operation will require a very big amount of energy, with power peaks up to $19000 \mathrm{~W}$. This represent the most challenging requirement for the Electrical Power Subsystem (EPS), for what concerns the handling of such power and the EMC related aspects.

The two satellites will operate in a Dawn/Dusk, sunsynchronous circular orbit.

Based on these operative constraints, the paper presents the way the CSG power generation and conditioning electronics have been designed and developed.

The power system chain to supply the SAR Antenna is made by the following equipment's:

- $10 * 24 \mathrm{Ah}$ Lithium-Ion (Li-Ion) battery modules.

- A Solar Array (SA) made by two different wings on each spacecraft.

- One Solar Array Drive Assembly (SADA) per SA wing.

- One Power Conditioning and Distribution Unit (PCDU) internally redounded.

- One SAR Antenna Power Distribution Unit (SPDU) providing an internal redundancy considering the acceptable performances graceful degradation of the SAR Antenna.

- 40 Tile Power Supply Units (TPSU). The SAR Tile is essentially a complex electronic box equipped with radiating panels that represents the elementary brick of the SAR antenna.

The paper provides a detailed description of all these equipment's of which Leonardo is the supplier, with the exception of the SADA and of the Battery modules (not under Leonardo responsibility) and, in particular, of how the extreme operative requirements of the mission are met.

It will be also shown how the design of the power conditioning electronic has taken advantage of the Leonardo (former Finmeccanica) heritage on main bus power conditioning for scientific platforms.

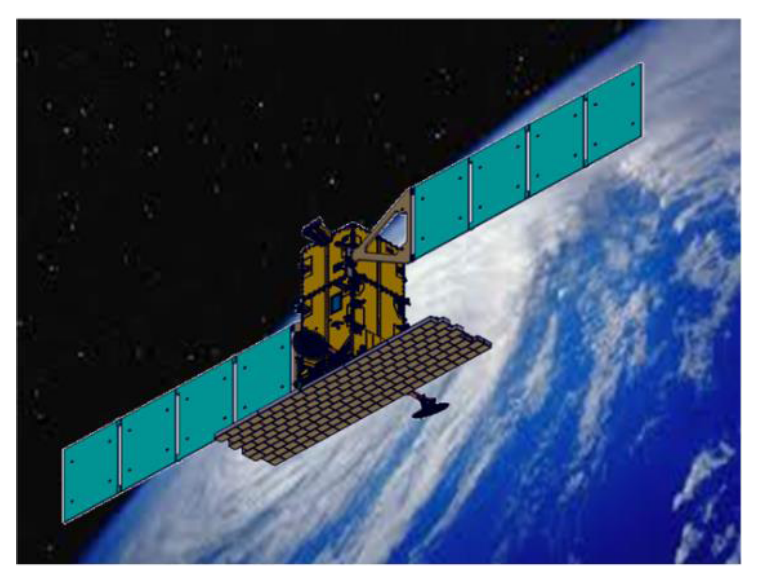

Figure 1. COSMO Second Generation satellite view. 


\section{EPS OVERVIEW}

The architecture of the Electrical Power Subsystem (EPS) of CSG is shown in Fig. 2.

The EPS of CSG provides two different busses:

- an unregulated $49 \mathrm{~V}$ to $67 \mathrm{~V}$ bus which supplies power peaks up to $19000 \mathrm{~W}$ during imaging. It also feeds the payload heaters located on the antenna.

- a regulated (P/F_PWR) $28 \mathrm{~V}$ bus which supplies up to $1800 \mathrm{~W}$, including distribution to Platform $(\mathrm{P} / \mathrm{F})$, to $\mathrm{P} / \mathrm{F}$ Heaters and to NEA and pyrotechnic devices.

The distribution to the Platform is realised via LCL protected lines which are allocated inside the PCDU.

The limitation and switching of the $\mathrm{P} / \mathrm{F}$ Heaters is performed inside SPDU.

The distribution to the SAR antenna is realised via LCL protected lines which are allocated inside the SPDU.

The power generator consists of a deployable Solar Array (SA) made of two wings of four panels each with a total area of $22 \mathrm{~m}^{2}$ and a peak power capability @ BOL of nearly $5 \mathrm{~kW}$.

The electrical energy is stored in 10 Lithium-Ion (LiIon) battery modules each of them with a capacity of 24Ah.

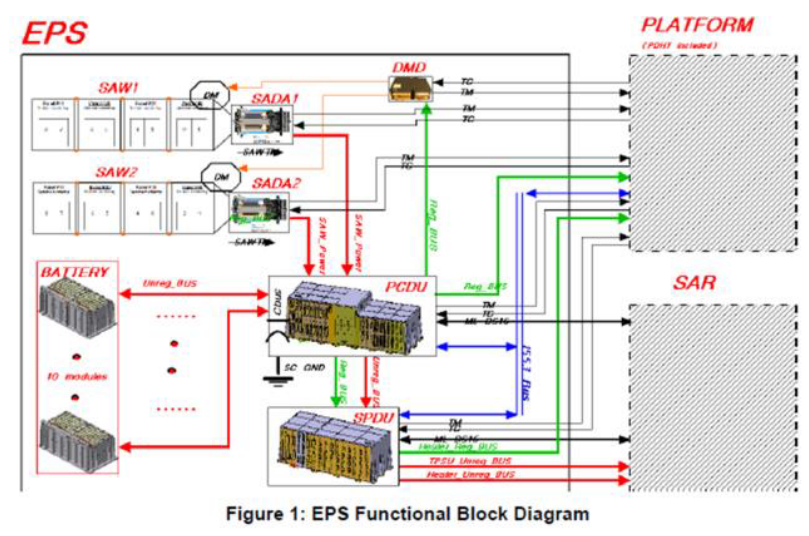

Figure 2: CSG EPS Configuration.

The EPS described in this paper is managed by Thales Alenia Space-Italy (TAS-I), which is leader of an industrial team composed of:

- Leonardo - Finmeccanica S.p.A. as responsible for the PVA, PCDU, SPDU and TPSU manufacturing.

- EnerSys ABSL as manufacturer of the batteries.

- RUAG Space as manufacturer of the SADAs.

TAS-I is also responsible of the whole spacecraft.

\section{POWER CONDITIONING AND DISTRBUTION UNIT (PCDU)}

The PCDU is part of the CSG EPS.

It houses in a single box the following functions:

- Power conditioning to provide the unregulated bus ranging from $49 \mathrm{~V}$ to $67 \mathrm{~V}$. It is based on 16 Sequential Switching Shunt Regulator (S3R) sections operating under control of a Battery Charge control Module (BCM) that controls the charge current released to the Batteries respect to the set Charge Current and End Of Charge Voltage (EOCV), operating in Constant Current (CC) or Constant Voltage (CV) modes depending on the inout power balance and performing the full batteries charge in tapered mode.

- Power conditioning to provide the $28 \mathrm{~V}$ regulated P/F_PWR bus, starting from the $49 \mathrm{~V}-67 \mathrm{~V}$ unregulated bus. It is based on 6 step-down DC/DC converters, operating under control of a Main Error Amplifier (MEA). The P/F_PWR bus is going to feed all the Platform $(\mathrm{P} / \mathrm{F})$ loads, the $\mathrm{P} / \mathrm{F}$ heaters located inside SPDU, as well as the NEA \& PYRO devices to release deployable appendages like Solar arrays and antennas.

- Distribution and protection via Latching Current Limiters (LCLs) and Retriggered Latching Current Limiters (R-LCLs) of the regulated power to the $\mathrm{P} / \mathrm{F}$ loads (essential ones connected to the R-LCL line).

- Distribution of regulated power to the protection lines for the $\mathrm{P} / \mathrm{F}$ heaters, located inside SPDU.

- Distribution and limitation of the current needed to actuate the NEA and PYRO devices necessary for the release of the satellite appendages.

- Reception of the Umbilical Supply, to perform nominal PCDU operation and battery maintenance, via external power sources during ground operations.

Fig. 3 shows the block diagram of the PCDU.

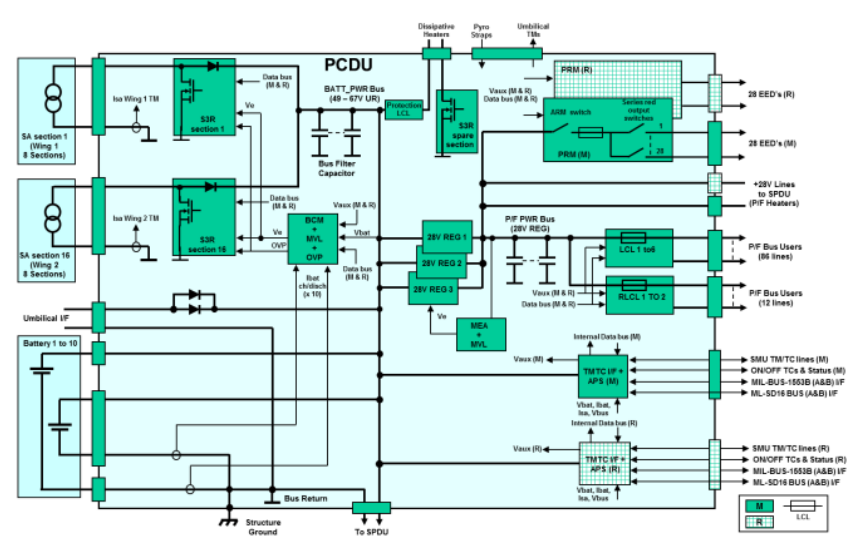

Figure 3. PCDU Block Diagram.

The input power will be provided by the SA during sunlight periods or by $10 \mathrm{Li}$-Ion battery modules during eclipses and when the SA power is lower than the power demand. The power generation consists of two SA wings made of 16 nominally identical sections.

The PCDU is sized to manage and provide during all mission phases a total peak output power of $19000 \mathrm{~W}$ towards the SAR antenna on the unregulated bus. This large amount of power will be released by the Solar Arrays according to their capability and to the current lightening conditions, integrated by the battery modules 
stored energy.

A suitable internal cabling (AWG8 wires are used) and metal bars system realise such power distribution with a voltage drop from battery power input interfaces to power output interfaces better than $0.4 \mathrm{~V}$.

\subsection{Unregulated Battery Bus Control}

The unregulated Battery Bus power is controlled in order to manage the Battery Charge. A triple redundant electronics (SPFF design) senses the actual Battery Voltage and the Battery Charge Current and compares them to the Battery Charge settings coming from 1553 bus, generating an Error Signal that commands the Sequential Switching Shunt Regulators (S3R) to:

- Open and release all the current available from the related Solar Array section to the Main Bus.

- Close a shunt element to exclude all the current available from the related Solar Array section from the Main Bus.

- Switch between the two above status' with a duty cycle proportional to the amount of the current necessary to reach the equilibrium in the charge control.

In this way the Battery charge is automatically performed in Constant Current (set current charge value) or in Constant Voltage (set battery voltage value). This latter realises a progressive (tapered) charge of the battery up to the final charge Voltage at zerocharge-current.

The S3R sections and the triple redundant control electronic are all fed by two internal power regulators (buck topology), working in hot redundancy, that realise a SPFF supply.

The power conditioning on the unregulated bus is completed by a $825 \mathrm{uF}$ capacitor bank made of 25 selfhealing PM907 type capacitors. The value has been defined as compromise between the specified maximum voltage ripple and the dynamic performances of the regulating voltage and current loops that perform the battery charge control.

The S3R sections have been designed with a single MOSFET to perform the shunting function and a single diode to connect the SA section to the Bus.

This choice allowed a great reduction in the physical space occupied by the S3R section, which was a critical aspect for the PCDU allocation inside the CSG spacecraft.

The critical failure of the diode in short circuit is controlled via an active electronic current limiter that contains the short current (both peak and limited value) and then opens in latched mode the MOSFET to remove the short circuit on Bus. The failed section is then permanently connected to the Main Bus.

A spare shunt, connected to the unregulated bus sinks exceeding power in case of loss of an S3R section fixed on bus, to avoid overvoltage.

No overvoltage protection has been foreseen inside
PCDU, as the SPFF control electronics, the constant presence of the battery and the spare shunt guarantee the nominal operation inside the specified battery voltage limits even in presence of a very low load.

\subsection{Regulated Platform Bus Control}

The PCDU is also sized to release up to $1800 \mathrm{~W}$ to the $\mathrm{P} / \mathrm{F}$ Bus, regulated at $28 \mathrm{~V}$ with a precision better than $\pm 0.5 \%$, in accordance with ECSS-E-ST-20C. This Bus feeds:

- all the platform loads, via LCL/R-LCL-protected lines.

- the $\mathrm{P} / \mathrm{F}$ heaters via non protected lines; LCL protection and switch interruption are realised inside SPDU

- the Electro Explosive Devices (EED) accounting the Non Explosive Actuators (NEA) and Pyro devices. PCDU supports two simultaneous NEA and Pyros fires with full power on $\mathrm{P} / \mathrm{F}$ lines in nominal operation. The EED can be actuated in sequence up to $6+6$ devices.

The $\mathrm{P} / \mathrm{F}$ Bus regulation is obtained via $6 \mathrm{DC}-\mathrm{DC}$ power regulators commanded in trans-conductance mode by a centralised, triple-redounded (SPFF) electronic.

The voltage control loop controls the current released by power modules outputs on a $3 \mathrm{mF}$ capacitor bank sensing the voltage across it. The capacitor bank is made of 30 self-healing PM90 type capacitors, The system meets the impedance requirement of ECSS-EST-20C.

The full power regulation is guaranteed by design also when only 5 DC-DC regulators are active as the power regulators have enough power capability to sustain $1 / 5$ of the bus full power.

\subsection{PCDU Power Distribution}

The power distribution of both the Busses inside PCDU is realised using metal power bars so to reduce at minimum the voltage drops and allow an easy, modular connection of all the modules whether they feed, control or supply the Busses themselves.

The distribution of the $\mathrm{P} / \mathrm{F}$ bus is performed by a number of protected lines belonging to the following two types:

- LCL for the non-essential loads that are disconnected after a HW-fixed delay when an overload appears at its output. Several sizes of LCL are present, identified by their maximum nominal current value. The intervention delay or Trip Off Time for each LCL type is sized tacking into account thermal stress considerations on the limiting elements (MOSFETs). The higher current LCL are obtained assembling in parallel basic LCL circuits; each LCL has its TM \& TC electronics. Each LCL is designed also to survive a multiple, repetitive 
overload (typically due to the presence of an Under Voltage Protection on the user side. LCLs can be externally commanded ON or OFF and give their status and the operating current as telemetries.

- R-LCL for the essential loads have the same principal characteristics as the LCLs, but they cannot be commanded ON or OFF via external commands; in case of over load they automatically re-start after a fixed time bouncing from the conduction and the limitation indefinitely. This bouncing can be excluded or re-activated via telecommands.

Both LCLs and R-LCLs have an input (on MB) UV protection with different thresholds (for the essential and non-essential loads); this protection is centralised at board level and disconnect simultaneously all the LCLs on that board.

The EED actuators perform the driving function of the Pyro or NEA devices feeding them with a fixed current for a fixed time duration. Their combination is sufficient to guarantee the device actuation. The actuator realises a triple barrier against unwanted activation:

- One HW interruption via a latching type relay (line ARM/DISARM).

- Two more HW levels to close the power line after the reception of:

$\checkmark$ A selection Telecommand that closes the addressed output electronic switch (series redounded to protect against the short circuit of one switch).

$\checkmark$ A fire Telecommand that closes the power line (common to up to 32 lines) via a non-latching relay, interrupting (commutation at no current) both hot and cold ends and via a series redounded Current Limiter, that sets the actuation current.

\subsection{PCDU Command and Monitor Interfaces}

The PCDU commanding and monitoring is provided through a Mil-BUS 1553 redounded interface and also through a Memory Load (ML) and Serial Data (SD) bus for some specific functions (Tile Control Unit LCL lines). For both the communication busses each terminal inside PCDU (Main and Redundant) can talk with both the main and redundant control units.

Several telemetries are available, giving a complete snapshot of the unit status during operation.

A number of temperature measurements (unconditioned thermistors) allow the thermal monitoring of the unit during AIT operation.

Fig. 4 shows a picture of the PCDU.

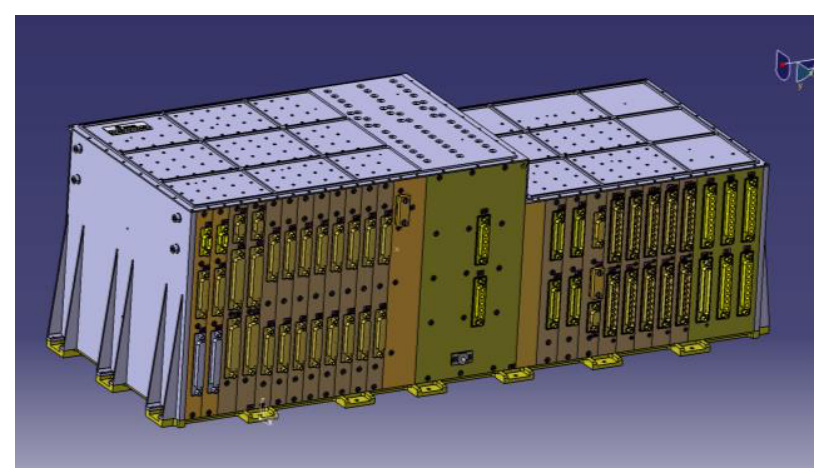

Figure 4: PCDU Box.

\subsection{PCDU Main Characteristics and budgets}

The main characteristics of the PCDU design are:

- Bus voltage: 49V-67V unregulated bus voltage.

- Unregulated (Battery) Bus power capability up to 19000W to SAR antenna (both Sun or Eclipse).

- Max Battery Charge Current ripple 5App.

- Battery Charge Voltage ripple depending on Battery and harness impedance. Maximum value is $5 \mathrm{~A}^{*} 0.062 \Omega=0.31 \mathrm{mVpp}$.

- Battery Charge Current in CC mode can be set via telecommand from $10 \mathrm{~A}$ to $80 \mathrm{~A}$; up to 498 different Current levels setting possible.

- Battery End Of Charge (EOC) Voltage in CV can be set from $62 \mathrm{~V}$ to $67 \mathrm{~V}$; up to 3641 different Current levels setting possible.

- SA current per section: 5A maximum in short circuit.

- 16 SA sections.

- P/F_PWR Bus maximum power: $1800 \mathrm{~W}$, including $850 \overline{\mathrm{W}}$ to $\mathrm{P} / \mathrm{F}$ heaters.

- 28V_REG module output power capability: $420 \mathrm{~W}$.

- PCDU power dissipation: < $300 \mathrm{~W}$ maximum at maximum power on both busses.

- TM/TC interface: MIL-1553 data bus + ML \& SD channels + direct lines.

- LCL classes: 1A, 2A, 3A, 5A, 7A, 10A, 12A.

- Total 86 LCL lines available.

- R-LCL class: $1 \mathrm{~A}$.

- Total 12 R-LCL lines available.

- Pyro/NEA Current: $5.5 \mathrm{~A} \pm 0.3 \mathrm{~A}$

- Pyro/NEA Pulse Duration: 40ms.

- Pyro/NEA shot capability: $6+6$ in $1.25 \mathrm{sec}$.

- $28+28$ Pyro/NEA lines available.

- Mass: $30.6 \mathrm{Kg}$.

- Dimensions: $740 \mathrm{~mm}$ x $297 \mathrm{~mm}$ x 250mm.

\section{SAR ANTENNA POWER DISTRIBUTION UNIT}

The SPDU is positioned in the EPS of CSG between PCDU and TPSUs, as it represents the distribution unit for the TPSU lines. 


\subsection{SPDU Architecture}

The SPDU box houses the following main functions:

- Distribution of unregulated power, fed by the PCDU, to:

$\checkmark$ Antenna loads.

$\checkmark$ SAR Tiles on-board heaters

- Distribution of regulated power (28V bus) to the Platform Heaters.

- Generation of Synchronism (sync) signals and their distribution to:

$\checkmark$ all the TPSUs. Two operative modes are possible for the sync signals:

- All in phase to each other

- All shifted with $36^{\circ}$ shift between adjacent signals.

$\checkmark$ SAR Electronics (SES) and SAR Antenna (SAS, including the TPSU units).

The Block Diagram in Fig. 5 summarises the functionality of SPDU.

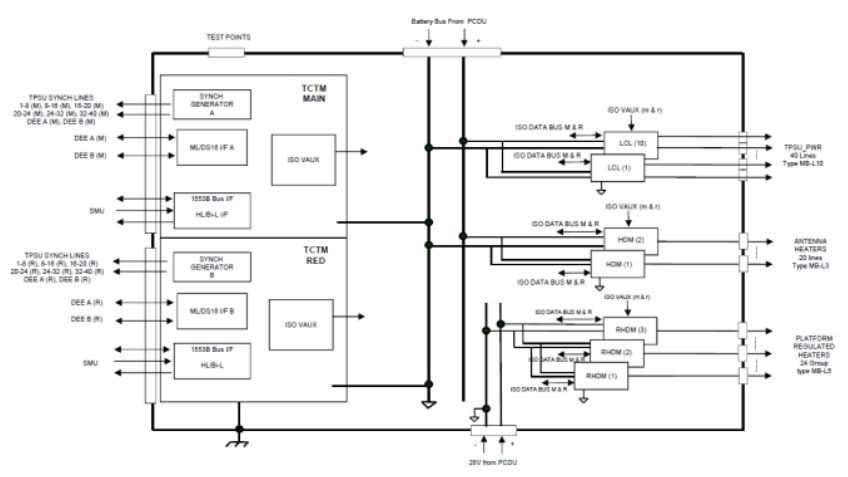

Figure 5. SPDU Block Diagram.

SPDU has the following two main tasks within the EPS:

- To provide Protection for all power outlets (LCL protected lines).

- To perform Filtering action for the Conducted Emission generated by TPSUs towards Batteries.

\subsection{Battery Bus Distribution}

Each power line towards TPSU inside the Antenna and each Antenna tile heater line are mainly composed of the series of a switch and a current limiter to protect bus against overload and to provide OFF protection to each Tile power line. Both the switch and the LCL are commanded ON/OFF via Memory Load telecommands that drive both the blocks. A delay in the actuation of the LCL ON/OFF with respect to the Switch ON/OFF can be added in the FPGA patterns telecommand.

The limitation current value of the power lines to TPSU has been defined in order to guarantee the operation of the TPSU and its correct switch-on (in-rush) in any specified condition, as well as to cope with thermal environment.

The limitation current value of the heater lines has been defined in order to cope with thermal environment.

Both these power lines implement a protection mechanism, managed by SW, that automatically disconnect the line in case of failure in linear mode of the switch element (MOS), as this dissipative failure cannot be sustained by the mechanical and thermal design. For the TPSU power lines it is possible to inhibit this protection intervention via dedicated 1553 Telecommand sent by on board computer.

On the TPSU power lines boards capacitive filtering are mounted, both on the input, which is common inside the SPDU) and on the output of each power line; the filtering is finalised to improve the EMC features of the chain (CE towards battery).

\subsection{Platform Bus Distribution}

The distribution of the $28 \mathrm{~V}$ regulated bus generated inside SPDU to the Platform heaters is organised in groups; each group, made of up to 5 heater lines is protected by a common current limiter.

Two sizes of heaters are fed; the groups have been organised so that the common current limiter, dimensioned to cope with thermal environment, can sustain the whole current required by the heaters belonging to that group.

All the distribution functions are managed in modular way, so that a suitable number of modules (boards) is used to meet the specified budgets.

\subsection{SPDU Command and Monitor Interfaces}

The SPDU commanding and monitoring is provided through a Mil-BUS 1553 redounded interface and also through a Memory Load (ML) and Serial Data (SD) bus for some specific functions.

SPDU implements a function (EQSOL) that, after the reception of a dedicated telecommand by on board computer, sequentially switches OFF (acting on LCL) all the logic group of TPSU_PWR lines (5 logic groups are foreseen) with a fixed delay $(6 \mathrm{~ms})$ between two consecutive power OFF.

The EQSOL function is aimed to perform at system level a quick switch-off of the Antenna in case of anomalous battery Under Voltage.

Fig. 6 shows a picture of the SPDU.

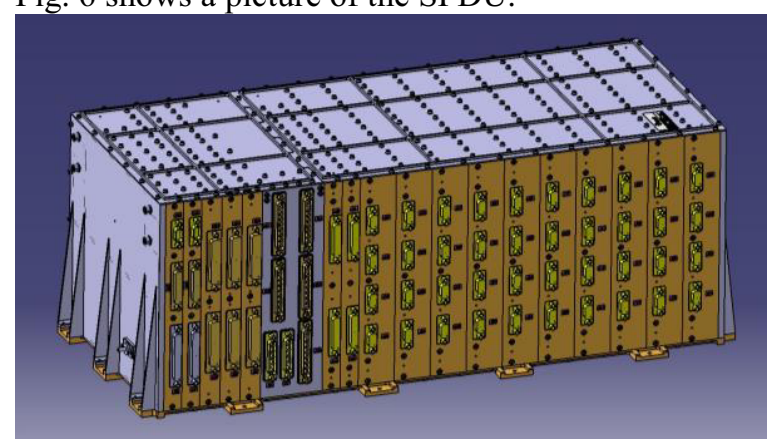

Figure 6: SPDU Box. 


\subsection{SPDU Main Characteristics}

The main characteristics of the PCDU design are:

- Bus voltage: $47 \mathrm{~V}-67 \mathrm{~V}$ unregulated bus voltage.

- Unregulated (Battery) Bus power capability up to $18700 \mathrm{~W}$ towards the SAR antenna.

- Total 40 Power lines to TPSU available.

- Unregulated (Battery) Bus power capability up to 2400W@67V towards the SAR Tile Heaters.

- Total 20 SAR Tiles Heater lines available.

- P/F_PWR Bus maximum power: $800 \mathrm{~W}$, towards P/F heaters.

- Total $108 \mathrm{P} / \mathrm{F}$ Heater lines available.

- $80 * 20 \mathrm{~W}$ lines

○ $28 * 60 \mathrm{~W}$ lines.

- SPDU power dissipation: $<300 \mathrm{~W}$

- 10 sync lines towards TPSU can be operated:

- All in phase

$\circ$ With $36^{\circ}$ phase shift between two neighbouring lines.

- 2 sync lines towards Electronics.

- TM/TC interface: MIL-1553 data bus + ML \& SD channels + direct lines.

- Two MOS protection SW loops to protect power lines against linear dissipative failures of the switches.

- EQSOL function to perform rapid removal of the power to the antenna in case of contingency.

\section{TILE POWER SUPPLY UNIT (TPSU)}

In order to properly supply the Antenna, a single Power Supply Unit for each Antenna Tile (TPSU) is foreseen. TPSU receives the input power directly from SPDU. Each protected power line in SPDU feeds a single TPSU.

TPSU generates the power lines necessary to supply the following items:

- 2 Electronic Front End (EFE), each composed of two benches of capacitors and 32 single $T / R$ modules.

- 1 True Delay Line (TDL)

TPSU is designed to generate seven different power supply lines, all regulated with an accuracy better than $3 \%$ EOL, galvanically isolated versus the primary power voltage.

The V1 and V2 lines supply the most powerful users, TX and RX module, and are generated via a dedicated regulator based on a full-bridge topology which uses the Synchronous Rectification technique.

This regulator manages up to $350 \mathrm{~W}$.

All the other Supply Voltages, V3 to V7 used to supply the T/R and EFE digital circuitry, are generated via a second multi-output regulator, based on a Push-Pull topology.

Four of the five output voltages are post regulated to meet the accuracy requirements.

No post regulation is provided for the EFE switch supply line, V7.

Fig. 7 shows a block diagram of TPSU.

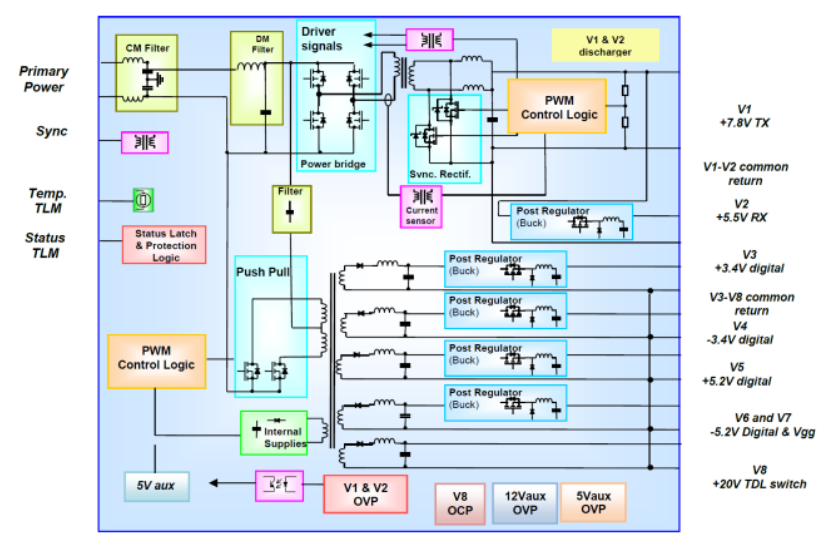

Figure 7. TPSU Block Diagram.

The total output power managed by TPSU is about $400 \mathrm{~W}$ with an efficiency better than $86 \%$.

The TPSU converters may operate in free running or synchronised by an external signal, TTL standard at $200 \mathrm{kHz}$, generated inside SPDU.

The secondary output rails grounding is provided with two separated return lines here below described:

- Lines V1 and V2 have a common return

- Lines from V3 to V7 have a common return.

The above common returns are referred to TILE chassis at user level, externally to TPSU

The TPSU switches automatically on when the Main Bus voltage is applied, according to SPDU dedicated LCL activation. The TPSU switches off when main bus voltage is removed by SPDU.

Each output line is able to supply the current to allow the fuse blowing in case of any fault at T/R level.

The TPSU mechanical design is based on a baseplate, as shown in Fig. 8, to provide thermal and mechanical interface with the Antenna tile mechanical structure where TPSU will be allocated.

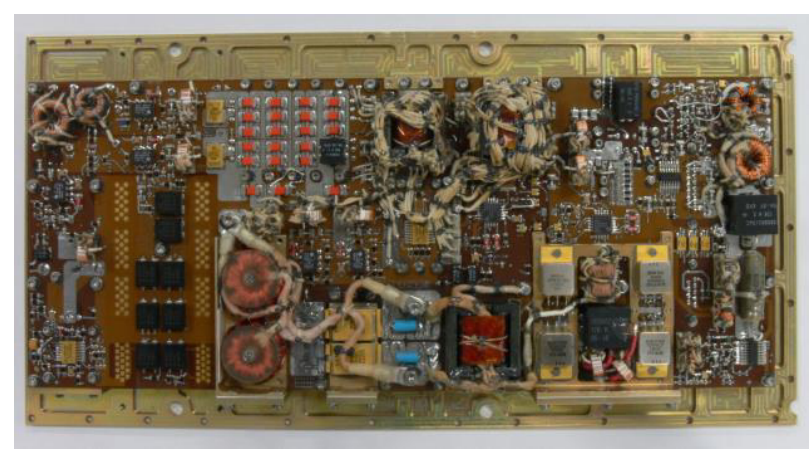

Figure 8. TPSU EM. 


\section{ELECTRO-MAGNETIC COMPATIBILITY (EMC) DESIGN APPROACH}

During imaging very high power changes cause a big perturbation on the power lines within the Pulse Repetition Frequency (PRF) range (from $500 \mathrm{~Hz}$ to $7000 \mathrm{~Hz}$ ).

The source of such perturbation are the TPSUs that directly feed the SAR antenna RF loads.

As the power of all the TPSUs is modulated synchronously, the resulting Conducted Emission (CE) in the PRF range on the power chain back to the Batteries is the sum of the CE of each TPSU.

Consequently, due to the battery impedance (maximum $\mathrm{DC}$ value is $62 \mathrm{~m} \Omega$ ), the resulting effect is a main bus voltage ripple that is present on all the Battery Bus users interface.

To validate the behaviour of all the chain both analyses and tests have been performed.

Fig. 9 shows the simulation of the CE injected by each TPSU line on the input Battery line, with maximum battery impedance. The noise at PRF is visible in the range $500 \mathrm{~Hz} \div 7.5 \mathrm{kHz}$.

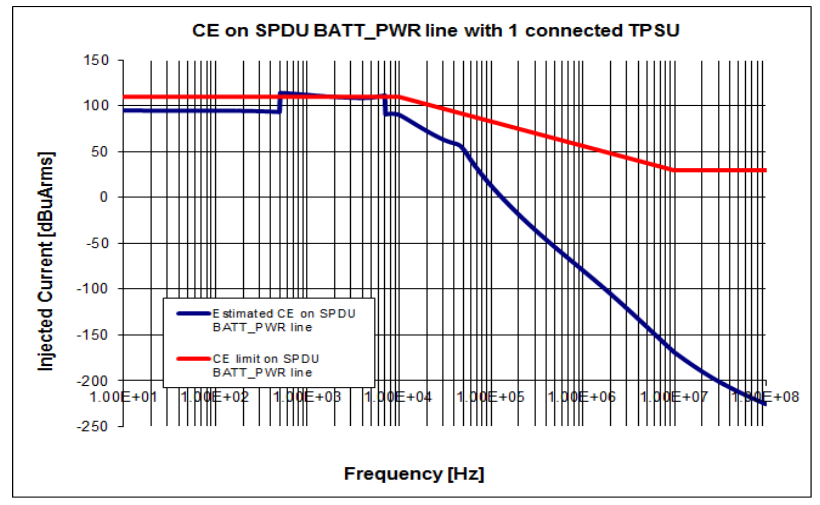

Figure 9. Simulated CE on SPDU Input line, 1 TPSU connected.

An EMC (CE) test has been performed at integrated level between one TPSU EM and one SPDU power module EBB.

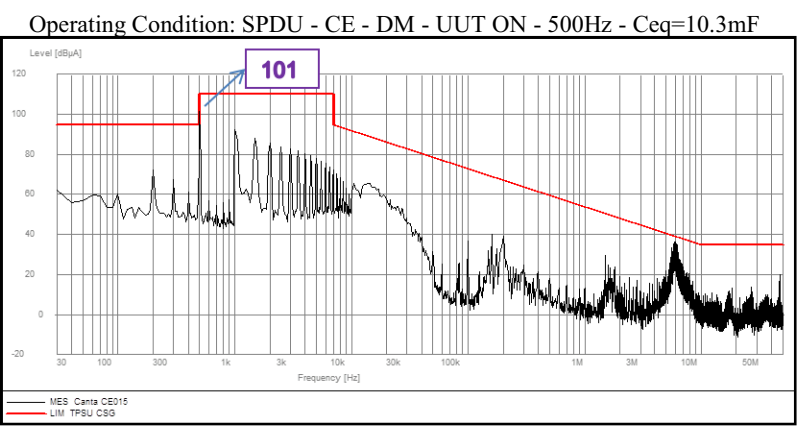

Figure 10. Measured CE on SPDU Input line, 1 TPSU connected (PRF at 500Hz).

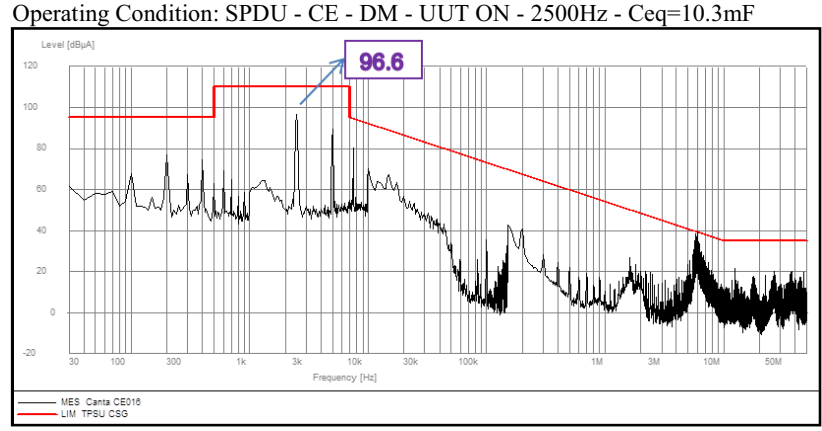

Figure 11. Measured CE on SPDU Input line, 1 TPSU connected (PRF at $2.5 \mathrm{kHz})$.

Fig. 10 and Fig. 11 summarise the test results at two PRF frequencies simulating the filtering effect of all the SPDU lines.

These results substantially confirm the values foreseen from the simulation.

On the basis of the simulation and test results (on one TPSU model) an analysis of the contribution of 40 TPSU simultaneously working has been performed with the Battery impedance as variable parameter. In order to verify complete behaviour of BTA impedance it has been considered the battery impedance variation dependence with:

- Aging

- Orbit

- Temperature

- State of Charge

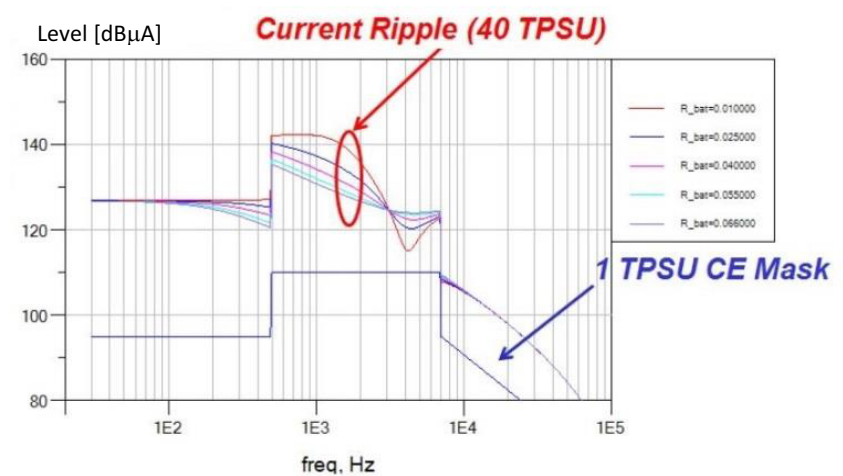

Figure 12. CE simulation on Battery line at varying Battery Impedance.

The S3R function inside PCDU has been sized and to sustain the (worst) specified disturbance at PRF without showing any susceptibility, e.g. S3R sections simultaneous commutation.

A set of test performed on an integrated EBB of the S3R sections of PCDU with a battery impedance of $62 \mathrm{~m} \Omega$, on the basis of the results summarised in Fig. 12, have also demonstrated the acceptability of such CE levels without susceptibility at power conditioning level. The PCDU SA regulating section $\mathrm{EBB}$, representative of the full S3R section, (including the Battery charge Control 
(BCM) module and the capacitor bank) has been submitted to a disturbance (injected on the load side) level of $138 \mathrm{~dB} \mu \mathrm{A}$, compatible with the above measured CE levels (at SPDU input) on the PRF range.

Fig. 13, Fig. 14 and Fig. 15, taken at the boundary frequencies of the PRF range and at $2 \mathrm{kHz}$, show the nominal behaviour of the S3R function; one section is switching with the expected proper ripple amplitude and frequency, without being affected by the noisy EMC environment.

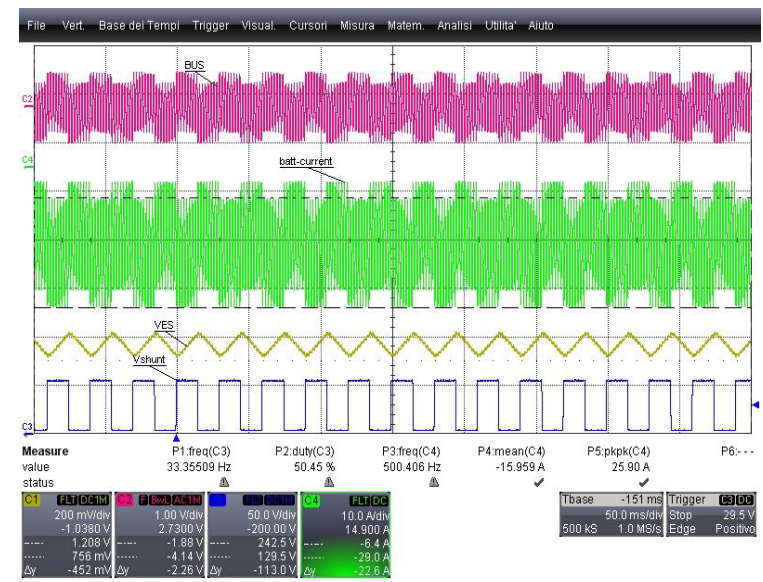

Figure 13. PCDU EMC_CS@500Hz.

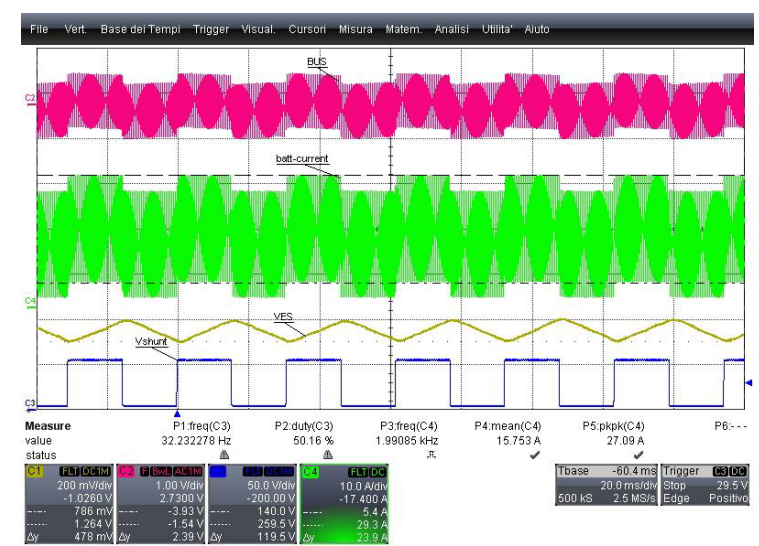

Figure 14.PCDU EMC_CS@2 KHz

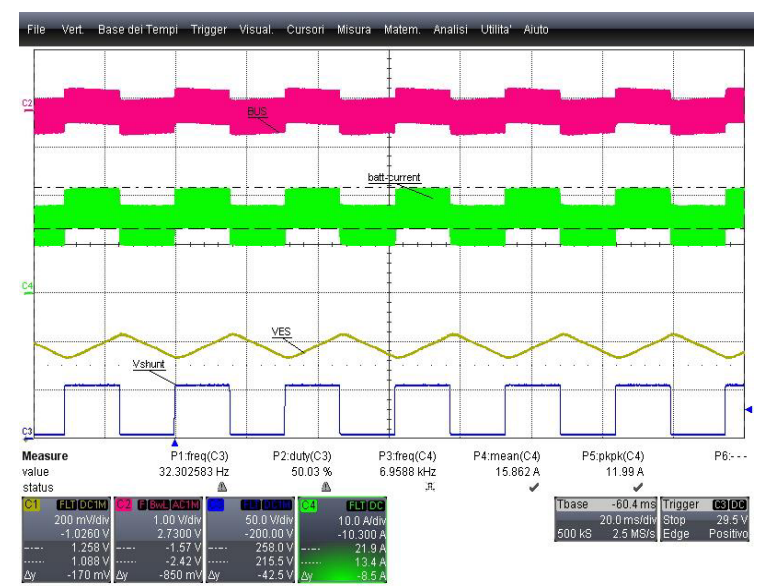

Figure 15.PCDU EMC_CS@7 $\mathrm{KHz}$

\section{PHOTOVOLTAIC ASSEMBLY}

The Photovoltaic Assembly is part of the SA, which design is driven by the mission requirements.

The Triple Junction 3G 28\% solar cells from AZUR space that represents the European consolidated technology for space-borne solar arrays, have been selected for this project.

The triangular silicon diode (AZUR space) is adopted for solar cell protection.

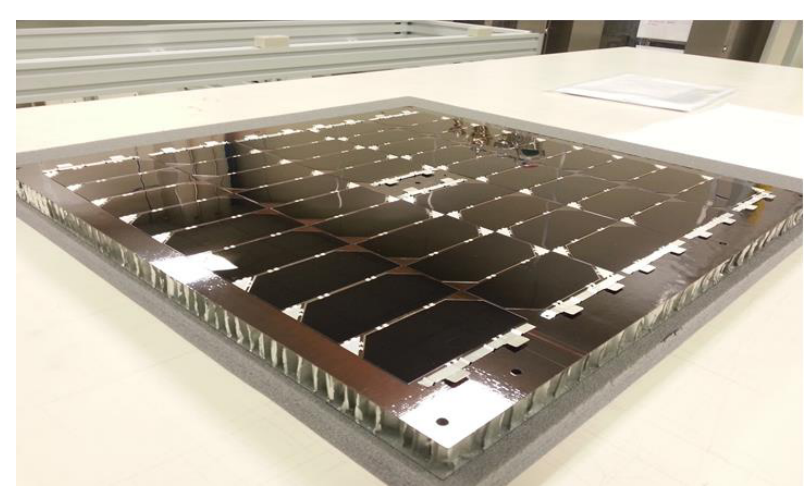

Figure 16. COSMO SG PVA DVT coupon.

The Solar Array for the Cosmo Second generation satellite is made of 2 identical wings of 4 panel each.

The PVA shall be divided into 2 electrical sections per panel.

The PVA S/C interface characteristics:

- Max open voltage of each section must be lower than 137 volts.

- Vmp shall be higher than 70V

- Voltage drop $<3 \mathrm{~V}$ average per wing at $70 \mathrm{~V}$ (avg $<$ $3.3 \mathrm{~V}$ for Imax BOL)

The Solar Array shall provide the following minimum power@70V:

- $3600 \mathrm{~W}$ at solar array level

- $450 \mathrm{~W}$ at panel level

- $500 \mathrm{~W}$ at panel level (stowed condition)

The PVA network is made of 2 electrical sections with 8 strings each (8p37s configuration).

Each string is protected by 2 blocking diodes grouped at section level into a single diode board.

The remaining factors and the temperature coefficients for Voc, Isc, Vmp and Pmax at the mission dose were calculated using a Matlab ${ }^{\circledR}$ interpolating function, which refer to the piecewise cubic Hermite interpolation.

The add-on mass of the PVA is less than $15 \mathrm{~kg}$.

\section{CONCLUSIONS AND FUTURE WORK}

CSG is a very demanding program that poses important challenges in terms of cost, mass and performances. 
In order to get the optimum choice between all these sometime conflicting requirements it has been selected a power conditioning architecture that is partly based on already qualified electronic modules.

Some design choices have privileged the simplicity as well as the cost and space saving approach. This is the case of the control of the SA power via an S3R approach, instead of the MPPT regulation approach. Other design, like the TPSUs have been developed ad hoc for the application, due to the specific requirements given by the upgraded SAR antenna, both in terms of electrical and of mechanical/thermal constraints.

\section{REFERENCES}

[1] L. Croci, G. Gianninoto, P. Galantini, E. Scorzafava, G. Daprati, M. Manfreda "COSMO SkyMed. The Electrical Power Subsystem Electromagnetic Compatibility issues", EDA Workshop on Power Supply and Energy Management for Defense Applications 13-14 June 2007.

[2] E. Scorzafava, F. Monaci, E. Zampolini et altri “ COSMO second generation: spacecraft design and technological challenge - Proceedings of EUSAR 2014; 10th European Conference on Synthetic Aperture Radar;

[3] Lazzeretti, L. Croci, G. Gianninoto, F.G. Re, "Spacecraft power conditioning and distribution: a modular and flexible approach for custom application," Proceedings of the 9th European Space Power Conference, 2014.

[4] F. Caltagirone, G. F. De Luca, E. Scorzafava, et altrii "COSMO-SkyMed di Seconda Generazione: Key Technological and Design Evolutions, and Related Potentialities" - Proceedings of 20th Ka and Broadband Communications, Navigation and Earth Observation Conference, Vietri, (2014).

\section{ACKNOWLEDGEMENTS}

The authors wish to thank all the colleagues that have contributed to the presented activity, with a particular thank to Luca Di Cola for his fundamental contribution. 